\title{
Quasiclassical Trajectory Study of Stereodynamics for Exchange Reactions $H^{\prime}\left(D^{\prime}\right)+H(D) S$
}

\author{
Bo Zhou and Qiang Wei* \\ School of Opoelectronic Information, Chongqing University of Technology, Chongqing \\ 400050, China
}

Received 1 January 2014; Accepted (in revised version) 20 February 2015

Published Online 1 March 2015

\begin{abstract}
Product Polarizations for the exchange reaction $\mathrm{H}^{\prime}+\mathrm{HS}$ and its isotopic variants on an accurate 1A'potential energy surface have been studied at collision energies of $10 \mathrm{kcal} / \mathrm{mol}$ by using the quasi-classical trajectory method (QCT). Product rotational angular momentum distributions $P\left(\theta_{r}\right)$ and $P\left(\phi_{r}\right)$ are calculated in the centerof-mass (CM) frame. Moreover, three generalized polarization-dependent differential cross sections (PDDCSs) are also computed. The results demonstrated that the isotopic effect displays sensitive effect on the product vector correlations. The reaction mechanism was discussed based on the properties of stereodynamics and the reactive trajectories.
\end{abstract}

PACS: 82.20.Fd, 71.15.Pd

Key words: stereodynamics; Isotopic Effects; Quasiclassical Trajectory; Exchange Reaction.

\section{Introduction}

The importance of vector correlations in reaction dynamics was first pointed out by Herschbach and coworkers based on the fact that chemical reactions are intrinsically anisotropic [1]. The vector and vector correlations include those of reagent $k-j, j-k-k^{\prime}$ correlations and those of product $k-j^{\prime}, k-k-j^{\prime}$ correlations, where $k / k^{\prime}$ and $j / j^{\prime}$ denotes the relative velocity of reagent/product and the reagent/product rotational angular momentum in the center of mass (CM) frame. The growing interest in the field is largely due to the advent of new experimental techniques and theoretical methods. Experimentally, the availability of new experimental methods has made it possible to measure and probe the product state-resolved rotational polarization and $k-k^{\prime}-j^{\prime}$ correlation, such

\footnotetext{
${ }^{*}$ Corresponding author. Email address: qiangwei@cqut.edu.cn (Q. Wei)
} 
as Doppler resolved laser induced fluorescence or resonance enhanced multiphoton ionization time-of-flight techniques. In order to easily interpret the vector correlations of the experimental measurements and clearly depict the corresponding stereodynamical picture, both the quasi-classical trajectory (QCT) [2-5] and the quantum scattering methods [6-7] have been developed to determine the product vector correlations. To date, the vector correlations has been widely and successfully studied in many stereodynamics calculations [8-15].

The gas-phase $\mathrm{S}\left({ }^{1} \mathrm{D},{ }^{3} \mathrm{P}\right)+\mathrm{H}_{2}$ reaction and its reverse reaction as well as the isotopic variants act as an important role in combustion and atmospherica chemistry, which have received considerable interest from last two decades. In previous work, many scalar properties such as product translational energy distribution [16], integral cross sections, differential cross sections and isotope branching ratio [17-23], potential energy surface [24-26], as well as the remarkable noadiabatic effect have been extensively studied. [2728] Recently, Guo and coworkers employed the QCT method to study the stereodynamics of abstract reaction $\mathrm{D}+\mathrm{DS}$ on the ${ }^{3} \mathrm{~A}^{\prime \prime}$ potential energy surface (PES), which has an energy barrier in the reaction path [29]. They have found that the product rotational angular momentum vectors are not only aligned, but also oriented. However, to the best of our knowledge, up to now there is still no correlative work to study the isotopic effect on stereodynamics properties of the $\mathrm{H}+\mathrm{HS}$ reaction on ${ }^{1} \mathrm{~A}^{\prime} \mathrm{PES}$. In this paper we mainly focus our attention on isotopic effect on stereodynamics properties of this reaction restricted on ${ }^{1} \mathrm{~A}^{\prime}$ PES.

\section{Theory}

The present calculations were performed by applying a standard QCT-stereodynamics procedure which has been successfully used to study a great deal of collision reactions. The center-of-mass (c.m.) frame is utilized in our calculations. The z-axis is parallel to the reagent relative velocity vector $k$, while the xz-plane (also called the scattering plane) contains $k$ and $k^{\prime}$ with $k^{\prime}$ on the $\mathrm{x} \geq 0$ half plane. The $\mathrm{y}$-axis is perpendicular to the scattering plane, $\theta_{r}$ is the angle between $k$ and $, j^{\prime}, \phi_{r}$ is the dihedral angle between the scattering plane and the plane containing $k$ and $j^{\prime}, \theta_{t}$ is the angle between $k$ and $k^{\prime}$. In the c.m. frame, the product rotational polarization can be depicted through angular distributions $P\left(\theta_{r}\right), P\left(\phi_{r}\right)$ and polarization-dependent generalized differential cross sections (PDDCSs). The product rotational polarization for the title reactions is investigated, using the stereo-QCT procedure which was developed by Han et al. [2-5]. Each reaction runs 100000 trajectories and the integration step size is set as $0.1 \mathrm{fs}$ to guarantee the conservation of total angular momentum and total energy. The calculations of the product rotational polarization with the initial rotational quantum number $j=0$ and initial vibrational quantum number $v=0$. The collision energy is $10 \mathrm{kcal} / \mathrm{mol}$ and the initial collision length is $15 \AA$ for each reaction.

The general theory of the product rotational polarization is standard, and here we 


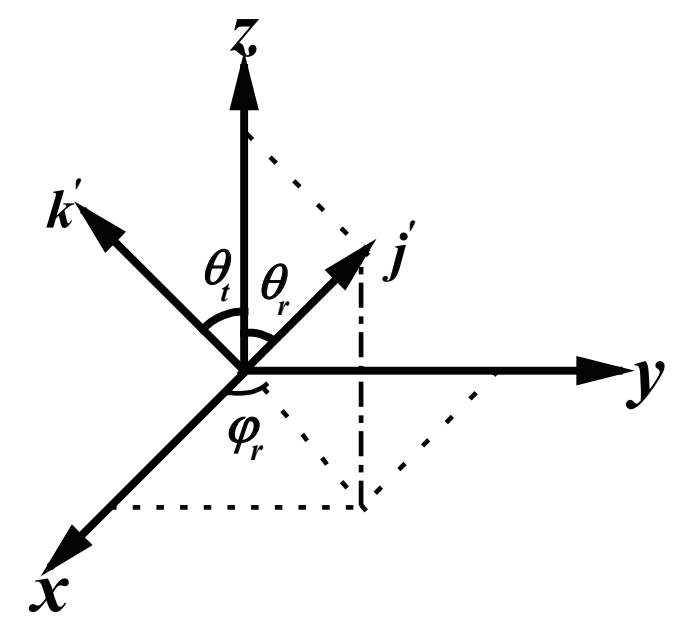

Figure 1: The center-of-mass coordinate system used to describe the $k, k^{\prime}$ and $j^{\prime}$ correlations.

only provide a simple description relevant to the present work. The reference frame used in this work is the center-of-mass (CM) frame, which is shown in Fig. 1. The reagent relative velocity vector $k$ is parallel to the $\mathrm{z}$ axis and the $\mathrm{x}-\mathrm{z}$ plane is the scattering plane containing the initial and final relative velocity vectors, $k$ and $k^{\prime}$. The angle $\theta_{t}$ is the so-called scattering angle between the reagent relative velocity and the product relative velocity. The angles $\theta_{r}$ and $\phi_{r}$ are the polar and azimuthal angles of the final rotational angular momentum $j^{\prime}$.

The distribution function $P\left(\theta_{r}\right)$ and the dihedral angle distribution function $P\left(\phi_{r}\right)$ described the $k-j^{\prime}$ and $k-k^{\prime}-j^{\prime}$ correlations, respectively. The function $P\left(\theta_{r}\right)$ can be expanded in a series of Legendre polynomials as

$$
\begin{aligned}
& P\left(\theta_{r}\right)=\frac{1}{2} \sum(2 k+1) a_{0}^{k} P_{k}\left(\cos \theta_{r}\right) \\
& \text { Where } a_{0}^{(k)}=\int_{0}^{\pi} P\left(\theta_{r}\right) P_{k}\left(\cos \theta_{r}\right) \sin \theta_{r} d \theta_{r}=\left\langle P_{k}\left(\cos \theta_{r}\right)\right\rangle
\end{aligned}
$$

The expanding coefficients $a_{0}^{(k)}$ are called the orientation ( $k$ is odd) or alignment ( $k$ is even) parameters. The dihedral angle distribution function $P\left(\phi_{r}\right)$ can be expanded in a series of Fourier series as

$$
P\left(\phi_{r}\right)=\frac{1}{2 \pi}\left(1+\sum_{\text {even }, n \geq 2} a_{n} \cos n \phi_{r}\right)+\sum_{\text {odd }, n \geq 1} b_{n}\left(\sin n \phi_{r}\right)
$$

Where

$$
\begin{aligned}
& a_{n}=2\left\langle\cos n \phi_{r}\right\rangle \\
& b_{n}=2\left\langle\sin n \phi_{r}\right\rangle
\end{aligned}
$$


In this calculation, $P\left(\theta_{r}\right)$ and $P\left(\phi_{r}\right)$ are expanded up to $k=18, \mathrm{n}=24$, respectively, which thereby showing good convergence.

The fully correlated CM angular distribution is written as the sum:

$$
P\left(\omega_{t}, \omega_{r}\right)=\sum_{k q} \frac{[k]}{4 \pi} \frac{1}{\sigma} \frac{d \sigma_{k q}}{d \omega_{t}} C_{k q}\left(\theta_{t}, \theta_{r}\right)
$$

where, $[\mathrm{k}]=2 \mathrm{k}+1, C_{k q}\left(\theta_{t}, \theta_{r}\right)=\sqrt{4 \pi /(2 k+1)} Y_{k q}\left(\theta_{r}, \phi_{r}\right)$ is modified spherical harmonics, $(1 / \sigma)\left(d \sigma_{k q} / d \omega_{t}\right)$ is a generalized PDDCSs and yields

$$
\begin{aligned}
& \frac{1}{\sigma} \frac{d \sigma_{k 0}}{d \omega_{t}}=0, k \text { is odd } \\
& \frac{1}{\sigma} \frac{d \sigma_{k q+}}{d \omega_{t}}=\frac{1}{\sigma} \frac{d \sigma_{k q}}{d \omega_{t}}+\frac{1}{\sigma} \frac{d \sigma_{k-q}}{d \omega_{t}}=0
\end{aligned}
$$

$\mathrm{k}$ is even and $\mathrm{q}$ is odd or $\mathrm{k}$ is odd and $\mathrm{q}$ is even.

$$
\frac{1}{\sigma} \frac{d \sigma_{k q-}}{d \omega_{t}}=\frac{1}{\sigma} \frac{d \sigma_{k q}}{d \omega_{t}}-\frac{1}{\sigma} \frac{d \sigma_{k-q}}{d \omega_{t}}=0
$$

$\mathrm{k}$ is even and $\mathrm{q}$ is even or $\mathrm{k}$ is odd and $\mathrm{q}$ is odd.

The PDDCS can write as

$$
\frac{1}{\sigma} \frac{d \sigma_{k q \pm}}{d \omega_{t}}=\sum_{k 1} \frac{k_{1}}{4 \pi} S_{k q \pm}^{k_{1}} C_{k_{1} q}\left(\theta_{t}, 0\right)
$$

Where $S_{k q \pm}^{k_{1}}$ is an expected value and write as

$$
S_{k q \pm}^{k_{1}}=\left\langle C_{k_{1} q}\left(\theta_{t}, 0\right) C_{k_{q}}\left(\theta_{r}, 0\right)\left[\left(-1^{q}\right) e^{i q \xi \phi} \pm e^{-i q \phi_{r}}\right]\right\rangle
$$

the angular brackets represent average over the all angles.

In the present work, we calculated the $k=0$ and $k=2,(2 \pi / \sigma)\left(\mathrm{d} \sigma_{00} / \mathrm{d} \omega_{t}\right),(2 \pi / \sigma)\left(\mathrm{d} \sigma_{20} / \mathrm{d} \omega_{t}\right)$, $(2 \pi / \sigma)\left(\mathrm{d} \sigma_{22+} / \mathrm{d} \omega_{t}\right)$ and $(2 \pi / \sigma)\left(\mathrm{d} \sigma_{21-} / \mathrm{d} \omega_{t}\right)$ named PDDCS ${ }_{00}$, PDDCS $_{20}$, PDDCS $_{22+}$ and PDDCS $_{21-}$ are calculated by using the computational code developed by Han and coworkers.

\section{Result and discussion}

As well known, $\mathrm{PDDCS}_{00}$ is proportional to the corresponding differential cross-section and describes the $k-k^{\prime}$ correlation. Fig. 2 displays four PDDCS $\mathrm{Po}_{00}$ of the $\mathrm{H}+\mathrm{HS}, \mathrm{H}+\mathrm{DS}$, $\mathrm{D}+\mathrm{HS}$ and D+DS reactions. As can be seen from Fig 2, the PDDCS $\mathrm{P}_{00} s$ of these four reactions show roughly symmetric behavior, with small sideways scattering and forward peaks which are similar in magnitude to the backward ones. This behavior is usually 


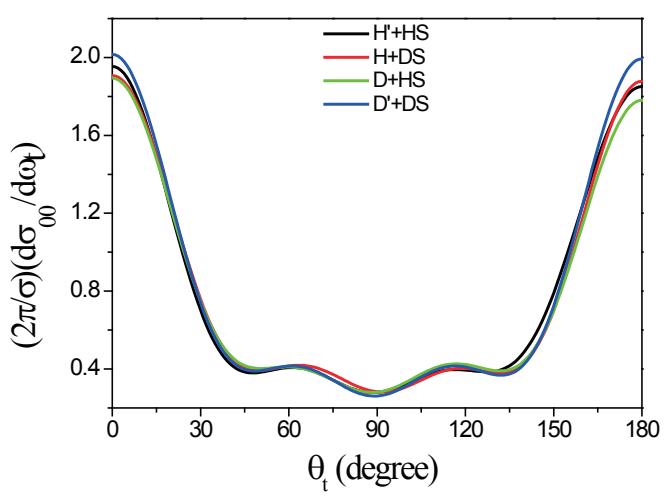

Figure 2: The QCT differential cross sections $\mathrm{PDDCS}_{00}$ of $\mathrm{H}^{\prime}\left(\mathrm{D}^{\prime}\right)+\mathrm{H}(\mathrm{D}) \mathrm{S}$ as a function of scattering angle.
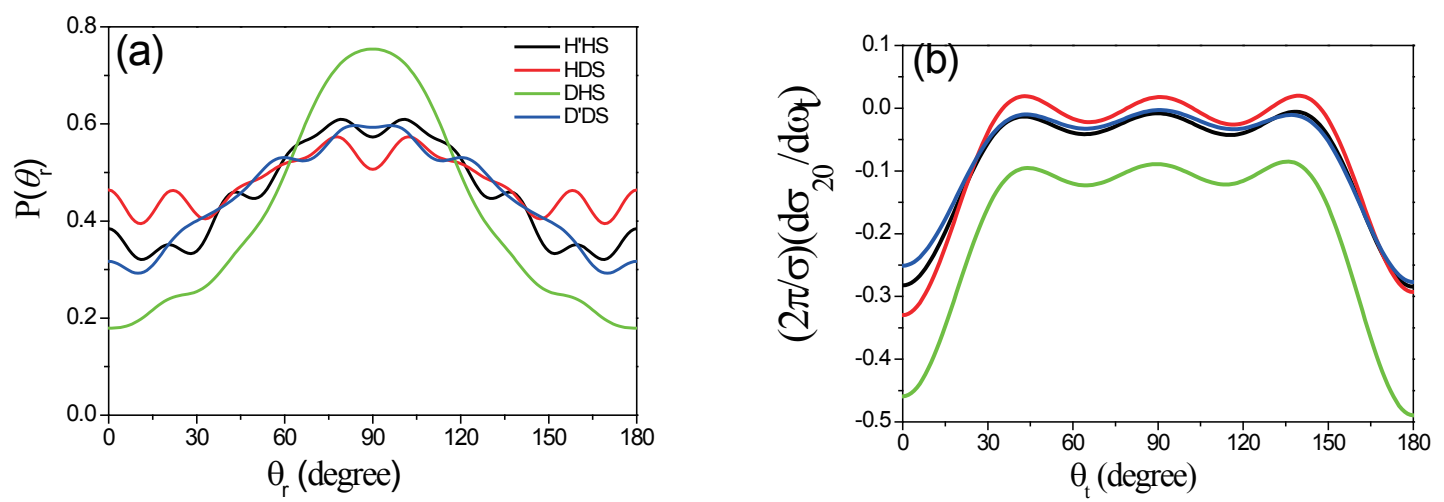

Figure 3: The distribution of $P\left(\theta_{r}\right)$ as a function of the polar angle $\theta_{r}$ and the distributions of $\mathrm{PDDCS}_{20}$ as a function of scattering angle $\theta_{t}$.

observed for a reaction involving a long-lived intermediate complex. It should be mentioned that in the case of $\mathrm{H}+\mathrm{HS}$ and $\mathrm{D}+\mathrm{HS}$ reactions, the distributions of $\mathrm{PDDCS}_{00}$ show little preference for forward scattering.

As mentioned above, the PDDCS $\mathrm{Po}_{00}$ only describes the $k-k^{\prime}$ correlation but not associated with the orientation and alignment of the product rotational angular momentum vector $j^{\prime}$. In order to further provide insight into the reactions, the correlation of the final rotational moment $j^{\prime}$ with respect to the relative velocity vector $\mathrm{k}$ are also calculated, which are presented by the distributions of $P\left(\theta_{r}\right)$. Fig. 3a presents the $P\left(\theta_{r}\right)$ distributions for exchange channel of H+HS, H+DS, D+ HS and D+ DS reactions at $10 \mathrm{kcal} / \mathrm{mol}$. Seen first from Fig. 3a, the distributions for these reactions are peaked at $90^{\circ}$ and symmetric with respect to $90^{\circ}$ due to the planar symmetry, which directly demonstrate that the product show preferentially polarization align the perpendicular direction to relative velocity $k$. The isotope effects on the $P\left(\theta_{r}\right)$ distributions can also be found from Fig. 3a. The alignment degrees for $\mathrm{H}+\mathrm{HS}$ and $\mathrm{D}+\mathrm{DS}$ reactions are almost equal to each other although a ' $\mathrm{V}$ ' 
shaped splitting has been found for $\mathrm{H}+\mathrm{HS}$ reaction. For $\mathrm{H}+\mathrm{DS}$ the distribution of $P\left(\theta_{r}\right)$ displays a distinct ' $\mathrm{V}$ ' shaped splitting and the peak is lower and broader than those of other three reactions. The highest and narrowest peak is found for $\mathrm{D}+\mathrm{HS}$ reaction among these $P\left(\theta_{r}\right)$ distributions. Another simple way to express the degree of polarization of $\mathbf{j}^{\prime}$ is the rotational alignment parameters. The distribution of the angular momentum $j^{\prime}$ of the product molecule is described by a function $f(\theta)$, where $\theta$ is the angle between $j^{\prime}$ and k. $f(\theta)$ can be represented by the Legende polynomial $f(\theta)=\sum a_{n} P_{n}(\cos \theta), \mathrm{n}=2$ indicates the product rotational alignment. The calculated product rotational alignment parameters are $-0.321,-0.301,-0.335$ and -0.323 corresponding to $\mathrm{H}+\mathrm{HS}, \mathrm{H}+\mathrm{DS}$, and D+ HS and $\mathrm{D}+\mathrm{DS}$ reactions, which is agreed with the $P\left(\theta_{r}\right)$ distributions. As a result, the isotope effects do affect the product polarization and it is showed that the smaller the mass of substituted atom than the incoming atom, the larger polarization for product.

The more detailed information about the product rotational angular momentum polarization can be analyzed from the PDDCS 20 calculated from formula 6. The PDDCS 20 is the expectation value of the second Legendre moment $\left\langle P_{2}\left(\cos \theta_{r}\right)\right\rangle$ and represents the product rotational angular polarization as functions of the scattering angle $\theta_{t}$. The allowed range for PDDCS 20 is from -0.5 to 1 and the more negative the value of PDDCS $_{20}$ the more the degree of the product rotational angular momentum $j^{\prime}$ is perpendicular to $k$. Therefore it is reasonable to compare their calculated values to the limiting values and thus have a clear idea of how strong is the polarization of the products. As shown in Fig. $3 b$, the PDDCS 20 is all negative for forward and backward scattered products for $\mathrm{H}+\mathrm{HS}$, $\mathrm{H}+\mathrm{DS}, \mathrm{D}+\mathrm{HS}$ and D+ DS reactions. This means that the rotational angular momentum $j^{\prime}$ of the forward and backward scattering products are preferentially polarized perpendicular to the reagent relative velocity $k$. However, for sideways scattering the values of PDDCS $_{20}$ are far away from the negative limiting but close to 0 , which indicates that the product rotational angular momentum polarization decreased for the sideway scattering product. So, it is clear that the angular momentum polarization are varied for different scattering angles, which can not be got form the $P\left(\theta_{r}\right)$ distributions.

The isotopic influence on PDDCS 20 can be analyzed for an exactly examination also from Fig. 3b. At scattering angle below $20^{\circ}$ the $\mathrm{PDDCS}_{20}$ for $\mathrm{H}+\mathrm{DS}$ reaction is more negative than those of $\mathrm{H}+\mathrm{HS}$ and $\mathrm{D}+\mathrm{DS}$ reaction but lower than those of $\mathrm{D}+\mathrm{HS}$ reaction. However for larger part of sideway scattering product, the $\mathrm{PDDCS}_{20}$ for $\mathrm{H}+\mathrm{DS}$ are almost zero but negative for $\mathrm{H}+\mathrm{HS}, \mathrm{D}+\mathrm{DS}$ and $\mathrm{D}+\mathrm{HS}$ reactions. Such distribution directly indicates that product angular momentum $j^{\prime}$ show somewhat isotropic for $\mathrm{H}+\mathrm{DS}$ but a little preferentially polarized perpendicular to the reagent relative velocity $k$. For backward products, the $\mathrm{PDDCS}_{20}$ is similar for $\mathrm{H}+\mathrm{HS}, \mathrm{D}+\mathrm{DS}$ and $\mathrm{D}+\mathrm{HS}$ reactions but is more negative for $\mathrm{D}+\mathrm{HS}$ reaction. It should be stressed that the $\mathrm{PDDCS}_{20}$ is more negative for $\mathrm{D}+\mathrm{HS}$ at 0-180 than those of other three reactions, which is agreement with the results of $P\left(\theta_{r}\right)$.

The above mentioned polarization only considers two vector correlations. In order to characterize fully the correlation between the three vectors $k, k^{\prime}$ and $j^{\prime}$ requires three angles, and the distributions $P\left(\theta_{r}\right)$, in the third angle, $\phi_{r}$, which defines the azimuthal 

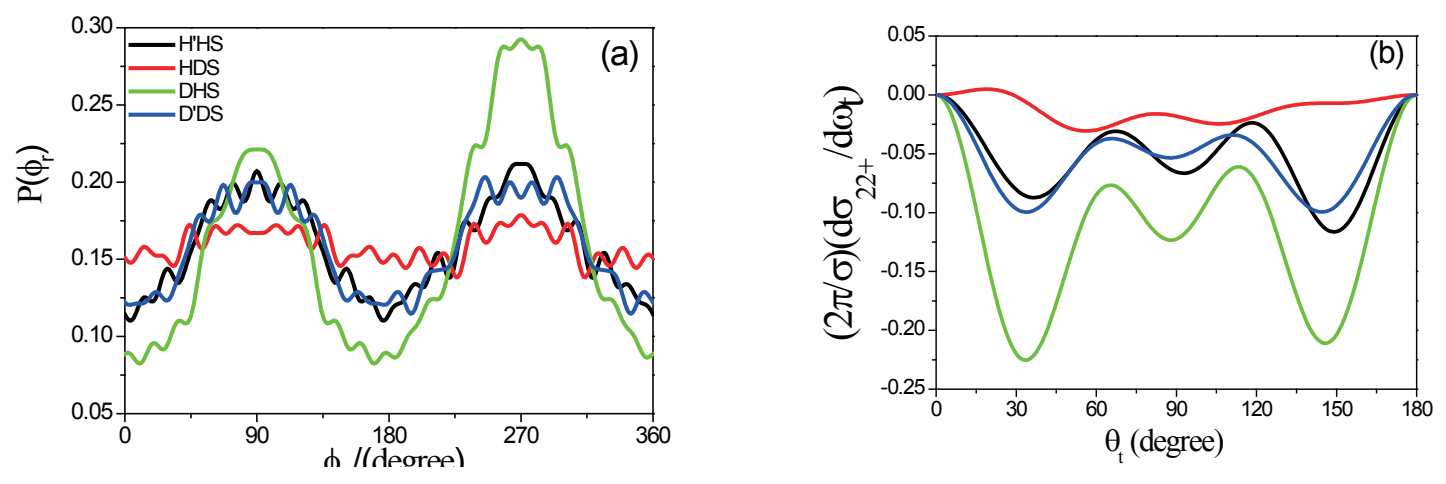

Figure 4: The distribution of $P\left(\phi_{r}\right)$ as a function of the polar angle $\phi_{r}$ and the PDDCS $22+$ as a function of scattering angle $\theta_{t}$.

angle between the vector $j^{\prime}$ and the $k-k^{\prime}$ scattering plane, are shown in Fig. 4a. Clearly, the distributions $P\left(\theta_{r}\right)$ can provide stereodynamical information on both product alignment and orientation. As can be seen from Fig. 4a, for $\mathrm{H}+\mathrm{HS}$ and D+ DS reactions, the appearance of the peaks at 90 degree and at 270 degree verified that the product rotational angular momentum vectors aligned along the y-axis. But the product orientation for these two reactions show no obvious preference along the negative or positive direction of the y axis, because of the almost symmetric feature of the two peaks located at 270 and 90 degrees. In contrast to these two reactions, for D+HS reaction, the $P\left(\phi_{r}\right)$ distribution reveals that the product rotational angular momentum is not only aligned along the $y$ axis but also oriented along the negative direction of the y-axis or is oriented such that $j^{\prime}$ points preferentially below the scattering plane due to the obvious asymmetric property of the two peaks located at 270 and 90 degrees (i.e. at angles $\phi_{r} \geq 180^{\circ}$ ). The most striking of the four distributions is that the obvious peaks at $\phi_{r}=90^{\circ}$ and $270^{\circ}$ are almost absent for $P\left(\phi_{r}\right)$ distribution of product come from the H+DS reaction. Such distribution indicates that product angular momentum has no preferentially alignment/orientation and is nearly isotropic in $\phi_{r}$.

Similarly, the detailed information about the $P\left(\phi_{r}\right)$ can also be obtained from the value of $\mathrm{PDDCS}_{22+}$. The positive or negative value of $\mathrm{PDDCS}_{22+}$ reveals the product alignment along $\mathrm{x}$-axis or along $\mathrm{y}$-axis. And the larger absolute value suggests the stronger alignment along the relevant axis. If the value is close to zero, there are no obvious alignment directions. From Fig. $4 b$, one can find that the values of PDDCS $_{22}$ for $\mathrm{H}+\mathrm{DS}$ are ranged from -0.03 to zero at all scattering angles, which further suggests that the product angular momentum for H+DS has no preferentially alignment/orientation with respect to $\mathrm{y}$-axis. For $\mathrm{H}+\mathrm{HS}$ and D+ DS reactions, one could find that the product alignment is along $y$-axis at the overwhelming number of scattering angles, which also could be observed through the $P\left(\phi_{r}\right)$ distributions in Fig $4 \mathrm{~b}$. Furthermore, the degree of polarization is different in different ranges of scattering angle for these two reactions. When the scattering angle is at about $26^{\circ}, 85^{\circ}$ and $145^{\circ}$, the intensity of polarization is 

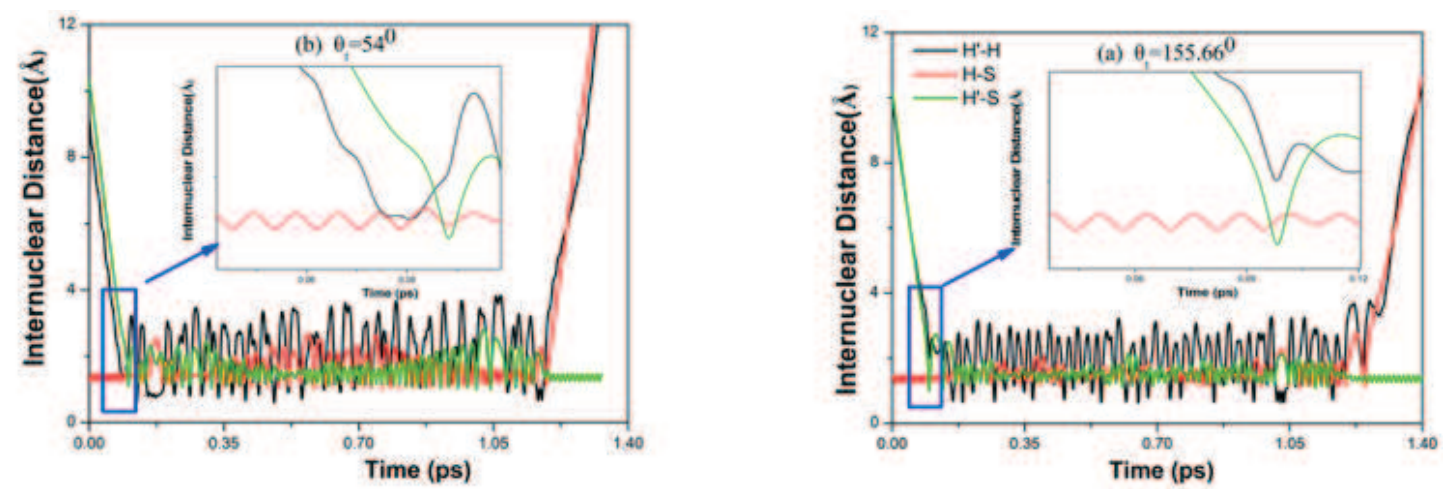

Figure 5: The internuclear distances of $\mathrm{H}^{\prime}-\mathrm{H}, \mathrm{H}^{\prime}-\mathrm{S}$ and $\mathrm{H}-\mathrm{S}$ as a function of propagating time on collision energy $\mathrm{E}_{\text {col }}=10 \mathrm{kcal} / \mathrm{mol}$, with scattering angle $\theta_{t}=155.66^{\circ}$ and $54^{\circ}$.
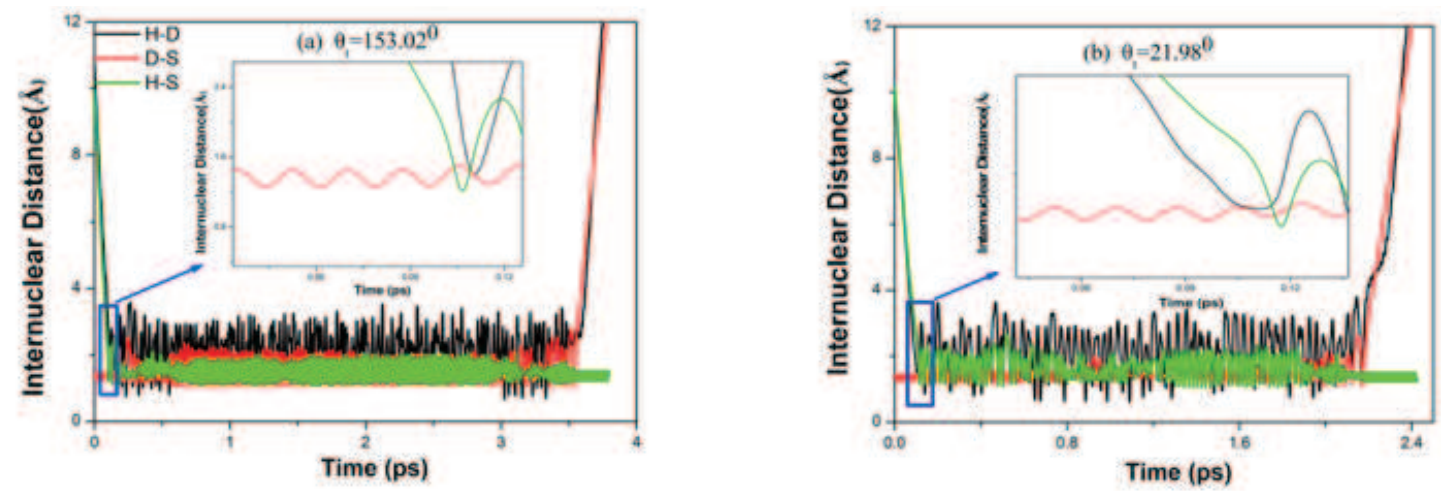

Figure 6: The internuclear distances of H-D, D-S and H-S as a function of propagating time on collision energy $\mathrm{E}_{\mathrm{col}}=10 \mathrm{kcal} / \mathrm{mol}$, with scattering angle $\theta_{t}=153.02^{\circ}$ and $21.98^{\circ}$.

stronger than those of other scattering angles. The overall trend for D+HS is similar to those of $\mathrm{H}+\mathrm{HS}$ and D+ DS but with more negative value of $\mathrm{PDDCS}_{22+}$.

As we know, the most distinguishing feature of the QCT method is to allow visualization and physical interpretation of the reaction dynamics. In Fig. 5-8, the internuclear distances for HHS, HDS, DHS and DDS as functions of propagation time at $10 \mathrm{kcal} / \mathrm{mol}$ are presented and the trajectories plotted here are from the product come from forward scattering, backward scattering and sideway scattering, respectively. It is useful to understand the product polarization by quantitative analyzing the internuclear distances of these reactions. For example, the HDS reaction, if the H-D distance raches, say, 1.5 before the H-S distance does, we say that $\mathrm{H}$ 'hits' $\mathrm{D}$ at 1.5. As can be seen from Fig. 5a, the direct approach of the $\mathrm{H}^{\prime}$ atom to the $\mathrm{S}$ end resulting in the formation of an $\mathrm{H}^{\prime} \mathrm{S}$ bond and the cleavage of the reactant HS bond yields direct mechanism trajectories. On the other 

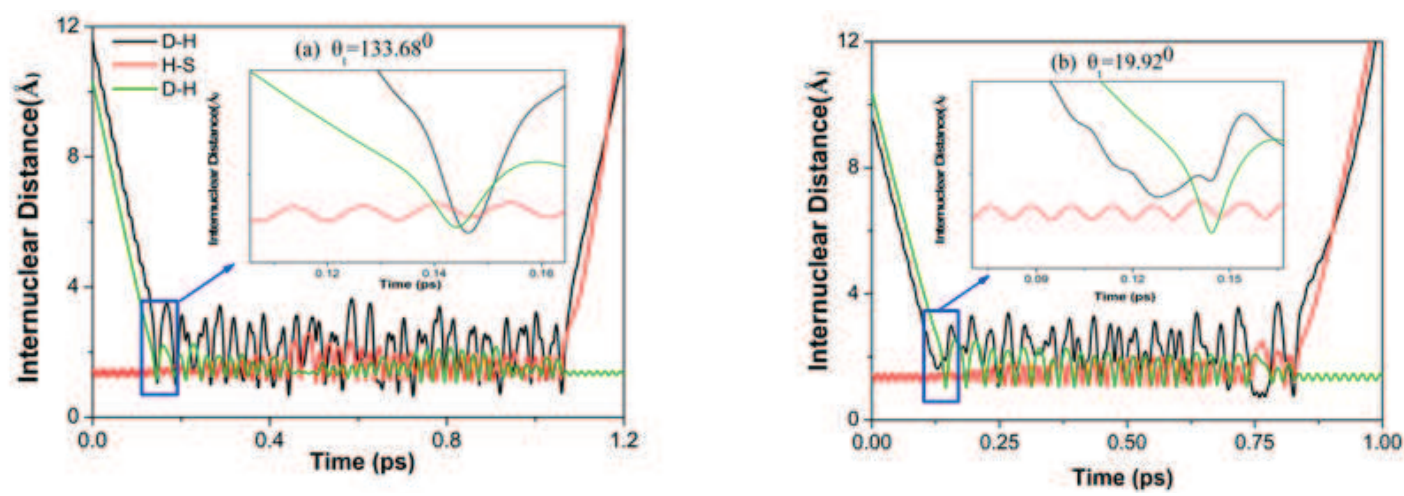

Figure 7: The internuclear distances of D-H, D-S and H-S as a function of propagating time on collision energy $\mathrm{E}_{\text {col }}=10 \mathrm{kcal} / \mathrm{mol}$, with scattering angle $\theta_{t}=133.68^{\circ}$ and $19.92^{\circ}$.
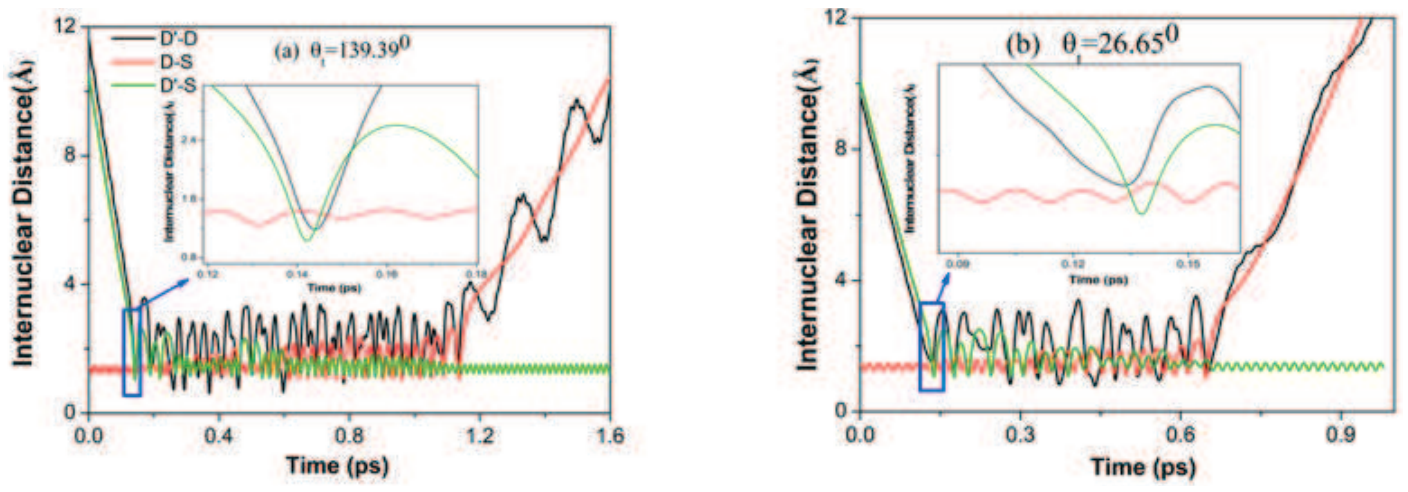

Figure 8: The internuclear distances of D'-H, D'-S and D-S as a function of propagating time on collision energy $\mathrm{E}_{\text {col }}=10 \mathrm{kcal} / \mathrm{mol}$, with scattering angle $\theta_{t}=139.39^{\circ}$ and $26.65^{\circ}$.

hand, non-direct mechanism trajectories are characterized by the attack of the $\mathrm{H}$ atom to the $\mathrm{H}$ end followed by a migration of the $\mathrm{H}^{\prime}$ atom to approach the $\mathrm{S}$ end resulting in the formation of the $\mathrm{H}^{\prime} \mathrm{S}$ product instead of the $\mathrm{H}^{\prime} \mathrm{H}$ one. This double microscopic mechanism has been also observed in recent QCT study of the $\mathrm{F}+\mathrm{HCl}$ reaction. As pointed by the author, the different microscopic mechanism corresponding to different orientation of the product in the reaction. This model can also be used in the present study. If the $\mathrm{H}^{\prime}$ atom first attack the $S$ end of the HS, the $j^{\prime}$ is preferred rotates clockwise in the scattering plane, leading to an orientation of $j^{\prime}$ preferentially along the -y direction. On the contrary, if the $\mathrm{H}^{\prime}$ atom first attack the $\mathrm{H}$ end of $\mathrm{HS}$, the $j^{\prime}$ is preferred rotates counterclockwise in the scattering plane, leading to a preferred orientation of $j^{\prime}$ along the $+y$ direction. Similar phenomenons have also been found for other three reactions. The alignment and orientation of $j^{\prime}$ for $\mathrm{H}^{\prime}+\mathrm{HS}$ reaction can be understood on the basis of the competition between the direct and non-direct mechanism. For $\mathrm{H}+\mathrm{HS}$ and D+DS reactions, both the direct and non-direct reaction mechanisms give the same contribution. However, the D+HS reac- 
tion is dominated by the direct mechanism leading to $\mathrm{H}^{\prime} \mathrm{S}$ rotational angular momentum orientation along the $-\mathrm{y}$ axis. For H+DS reaction, the direct and non-direct mechanisms also play the same role in the reaction, but the apparent difference is the lifetime of the complex formed in the reaction. It is clear that the lifetime of the formed complex for $\mathrm{H}+\mathrm{DS}$ is much larger than those of other three reactions, which is long enough for vibration and rotation of complex. As a result, the $j^{\prime}$ for H+DS reaction loses memory of their initial direction and randomize the direction of rotational angular momentum easier than those of other three reactions. Hence the orientation and alignment of the H+DS reaction is the weakest among these three reactions.

\section{Conclusions}

The stereodynamical calculations of the isotopic reactions $\mathrm{H}(\mathrm{D})+\mathrm{H}$ (D) S were performed on the ${ }^{1} \mathrm{~A}^{\prime} \mathrm{PES}$ at $10 \mathrm{kcal} / \mathrm{mol}$ by means of QCT method. The $P\left(\theta_{r}\right), P\left(\phi_{r}\right), P\left(\theta_{r}, \phi_{r}\right)$ as well as four normalized PDDCSs were calculated and discussed. It is found that the PDDCS 00 was symmetric forward and backward scattering for $\mathrm{H}+\mathrm{DS}$ reaction, but preference for forward scattering for $\mathrm{H}+\mathrm{HS}, \mathrm{D}+\mathrm{DS}$ and $\mathrm{D}+\mathrm{HS}$ reactions. For these four reactions the rotational angular momentum vector $j^{\prime}$ of product $\mathrm{H}_{2}$ (HD) was preferentially aligned perpendicular to $k$. The product rotational angular momentum vector $j^{\prime}$ of $\mathrm{H}+\mathrm{HS}, \mathrm{D}+\mathrm{DS}$ and $\mathrm{D}+\mathrm{HS}$ reactions is not only aligned, but also oriented along the direction perpendicular to the scattering plane. However the product rotational angular momentum vector $j^{\prime}$ of $\mathrm{H}+\mathrm{DS}$ is only align the perpendicular direction to relative velocity $k$ but show isotropic with respect to the scattering plane. The reaction mode was discussed through the reactive trajectories and two reaction mechanisms are confirmed. For $\mathrm{H}+\mathrm{HS}, \mathrm{H}+\mathrm{DS}$ and $\mathrm{D}+\mathrm{DS}$ reactions both the direct and non-direct reaction mechanisms give the same contribution but with the longest lifetime for metastable intermediate of $\mathrm{H}+\mathrm{DS}$. For D+HS reaction is dominated by the direct mechanism leading to H'S rotational angular momentum orientation along the $-y$ axis.

Acknowledgments This work is supported by the Natural Science Foundation of China (No.11204392 and No.11047125) and Scientific and Technological Research Program of Chongqing Municipal Education Commission (Grant No. KJ1400920). The author also acknowledges Professor Keli Han for providing the QCT code of stereodynamics.

\section{References}

[1] G. M. Mcclell, D. R. Herschbach, J Phys Chem A. 83 (1979) 1445.

[2] K. L. Han, G. Z. He, N. Q. Lou, Chin J Chem Phys. 2 (1989) 323.

[3] K. L. Han, G. Z. He, N. Q. Lou, Chin Phys Lett. 4 (1993) 517.

[4] R. J. Li, K. L. Han, F. E. Li, G. Z. He, N. Q. Lou, Chem Phys Lett. 220 (1994) 281.

[5] K. L. Han, G. Z. He, N. Q. Lou, J Chem Phys.105 (1996) 8699.

[6] M. P. Miranda, D. C. Clary, J Chem Phys. 106 (1997) 4509.

[7] M. P. Miranda, D. C. Clary, J. F. Castillo, D. E. Manolopoulos, J Chem Phys. 108 (1998) 3142. 
[8] M. D. Chen, K. L. Han, N. Q. Lou, J Chem Phys. 118 (2003) 4463.

[9] Q. Wei, X. Li, T. Li, Chemical Physics.368 (2010) 58.

[10] D. Fazio, J Chem Phys. 125 (2006) 133109.

[11] N. Balucani, G. Capozza, E. Segoloni, R. Bobbenkamp, P. Casaveechia, T. Gonzalez-Lezana, E. J. Rackham, L. Bãnares, and J. F. Aoiz, J. Chem. Phys. 122, 234309 (2005).

[12] F. J. Aoiz, V. J. Herrero, M. P Mirandac, Phys Chem Chem Phys. 9 (2007) 5367.

[13] X. H. Li, M. S. Wang, I. Pino, C. L. Yang, L. Z. Ma, Phys Chem Chem Phys. 11 (2009) 10438.

[14] W. Q. Zhang, S. L. Cong, C. H. Zhang, X. S. Xu, M. D. Chen, J Phys Chem A. 113 (2009) 4192.

[15] T. S. Chu, J Comput Chem. 31 (2009) 1385.

[16] Y. Inagaki, S. M. Shamsuddin, Y. Matsumi, M. Kawasaki, Laser Chem. 14 (1994) 235.

[17] S. H. Lee, K. Liu, Chem Phys Lett. 290 (1998) 323.

[18] S. H. Lee, K. Liu, Appl Phys B Lasers Opt .71 (2000) 627.

[19] S. H. Lee, K. Liu, J Phys Chem A. 102 (1998) 8637.

[20] L. Mouret, J. M. Launay, M. T. Dunseath, K. Dunseath, Phys Chem Chem Phys. 6 (2004) 4105.

[21] H. Yang, K. L. Han, G. C. Schatz, S. C. Smith, M. Hankel, Phys Chem Chem Phys. 12 (2010) 1271.

[22] H. Yang, K. L. Han, G. C. Schatz, S. H. Lee, K. Liu, S. C. Smith, M. Hankel, Physical Chemistry Chemical Physics. 11 (2009) 11587.

[23] S. J. Lv, P. Y. Zhang, G. Z. He, Chin Phys Lett. 29 (2012) 073401.

[24] S. Z. Alexander, M. M. Alexander, D. C. Sheng, R. T. Skodje, J Chem Phys. 114 (2001) 320.

[25] S. J. Lv, P. Y. Zhang, K. L. Han, G. Z. He, J Chem Phys. 136 (2012) 094308.

[26] T. S. Ho, T. Hollebeek, H. Rabitz, J Chem Phys. 116 (2002) 4124.

[27] B. Maiti, G. C. Schatz, G. Lendvay, J Phys Chem A. 108 (2004) 8772.

[28] T. S. Chu, K. L. Han, G. C. Schatz, J Phys Chem A. 111 (2007) 8286.

[29] Y. H. Guo, F. Y. Zhang, H. Z. Ma, Chin Phys B. 22 (2013) 053402. 